\title{
Casticin suppresses the carcinogenesis of small cell lung cancer H446 cells through activation of AMPK/FoxO3a signaling
}

\author{
QIAN GONG $^{1 *}, \mathrm{XIAOZHENG} \mathrm{CAO}^{2 *}$, JIANGUO CAO $^{3,4}, \mathrm{XIAOHONG} \mathrm{YANG}^{3,4}$ and WENBIN ZENG ${ }^{2}$ \\ ${ }^{1}$ Pharmacy Department, Hunan Cancer Hospital/The Affiliated Cancer Hospital of Xiangya School of Medicine; \\ ${ }^{2}$ School of Pharmaceutical Sciences, and Molecular Imaging Research Center, Central South University; \\ ${ }^{3}$ Department of Pharmaceutical Science, Medical College, Hunan Normal University; \\ ${ }^{4}$ Key Laboratory of Study and Discovery of Small Targeted Molecules of \\ Hunan Province, Changsha, Hunan 410013, P.R. China
}

Received January 10, 2018; Accepted June 12, 2018

DOI: $10.3892 /$ or.2018.6547

\begin{abstract}
Casticin, a natural polymethoxyflavone isolated from A. annua, V. trifolia, and V. agnus-castus induces apoptosis in cancer cells by activating FoxO3a. However, whether casticin inhibits in vitro carcinogenesis and cancer stem cell (CSC) characteristics, and whether casticin activates FoxO3a in small cell lung cancer (SCLC) cells remain unclear. We here demonstrated that casticin decreased sphere- and colony-formation capabilities, and downregulated UPAR and CD133 in second-generation spheres, which were considered as lung cancer stem-like cells (LCSLCs), from SCLC H446 cells, in a concentration-dependent manner. In addition, casticin dose-dependently elevated the phosphorylation levels of AMPK and ACC, and reduced p-FoxO3a expression. The above effects were attenuated by AMPK knockdown with small interfering RNAs (siRNAs). FoxO3a silencing resulted in decreased protein expression of FoxO3a, increased in vitro carcinogenesis and CSC characteristics, with no appreciable effects on AMPK and ACC phosphorylation, and displayed similar activities to those neutralizing the effects of casticin on in vitro carcinogenesis and CSC characteristics. These findings reveal a novel mechanism for regulating AMPK/FoxO3a signaling in response to casticin, suggesting a new strategy for SCLC therapy by targeting cancer stem-like cells.
\end{abstract}

Correspondence to: Dr Jianguo Cao, Department of Pharmaceutical Science, Medical College, Hunan Normal University, 371 Tongzipo Road, Changsha, Hunan 410013, P.R. China

E-mail: caojianguo2005@126.com

*Contributed equally

Key words: small cell lung cancer, cancer stem cells, casticin, AMPK, FoxO3a

\section{Introduction}

Small cell lung cancer (SCLC), a poorly differentiated and highly aggressive tumor, constitutes approximately $15 \%$ of all lung cancers (1). SCLC patients often present with metastasis at diagnosis, ruling out surgery as a treatment option (2). There is currently no approved targeted drugs for SCLC, and no effective method for early diagnosis is available; meanwhile, most patients treated with conventional therapies, including chemotherapy and radiotherapy, show recurrence after a short period of time, which results in poor patient prognosis (3). To improve the survival of patients with SCLC, developing new and efficacious candidate agents is urgently required.

Emerging evidence suggests that cancer stem cells (CSCs), a subpopulation of tumor cells, have the properties of self-renewal, heterogeneous progeny, drug-resistance, and carcinogenesis in vitro and in vivo $(4,5)$. Multiple SCLC characteristics, such as aggressiveness and high metastatic potential, suggest that this cancer could be enriched in CSCs (6). Furthermore, drug resistance may be attributable to the occurrence of a CSC subpopulation in SCLC (6). Therefore, a therapeutic strategy targeting CSCs may help cure malignant tumors, including SCLC (7).

FOXO3a is considered an evolutionarily conserved transcription factor involved in various cellular processes, including cell cycle arrest, DNA repair and tumor suppression (8). In cancer progression, FOXO3a inhibition stimulates cell transformation and angiogenesis (9). Conversely, FOXO3a overexpression suppresses cancer cell growth, induces apoptosis, and reduces tumor size by regulating downstream effectors (10). These findings suggest a tumor suppressor role for FOXO3a, which could constitute a potential target for cancer treatment. Our previous findings demonstrated that casticin, a natural polymethoxyflavone isolated from A. annua, $V$. trifolia, and V. agnus-castus that has been widely used in traditional Chinese medicine as an anti-inflammatory drug for thousands of years, not only induces growth suppression, apoptosis and cell cycle arrest in hepatocellular carcinoma (11) and breast cancer cells (12), but also promotes apoptosis in ovarian cancer SKOV3 cells (13) through FOXO3a activation. However, whether casticin inhibits in vitro carcinogenesis and 
CSC characteristics in the SCLC H446 cell line, and activates FoxO3a remains unclear.

Yung et al (9) demonstrated that AMPK activation inhibited cervical cancer cell growth through AKT/FOXO3a/FOXM1 signaling. Meanwhile, Shrestha et al (14) reported that the AMPK/FoxO3A axis plays a critical role in the antiproliferative effects of adiponectin in cancer cells. Furthermore, Sato et al proposed that metformin efficaciously eliminates glioma stem cell-like cells by activating FOXO3 via AMPK (8). Moreover, Zhao et al provided evidence that GL-V9, a new synthetic flavonoid derivative, improved the state of animals with DSS-induced colitis from oxidative stress by upregulating Trx-1 via activation of the AMPK/FOXO3a pathway (15). However, whether the anticancer effects of casticin involve AMPK/FoxO3a signaling remains undefined.

The present study showed that casticin inhibited in vitro carcinogenesis and CSC characteristics in LCSCs derived from the H446 cell line, as demonstrated by sphere- and colony-formation assays, as well as western blot analysis. Mechanistically, the effects of casticin were partly associated with activation of AMPK/FoxO3a signaling. These findings suggest that casticin may be used as a novel candidate agent for SCLC treatment targeting lung cancer stem-like cells.

\section{Materials and methods}

Cell culture and reagents. The human small cell lung cancer NCI-H446, H209 and H69 cells were obtained from the Cell Bank of the Chinese Academy of Sciences (Shanghai, China), and maintained in Dulbecco's modified Eagle's medium (DMEM, Life Technologies, Grand Island, NY, USA) supplemented with $10 \%$ fetal bovine serum (FBS) (Invitrogen, Shanghai, China), $100 \mathrm{U} / \mathrm{ml}$ penicillin and $100 \mathrm{U} / \mathrm{ml}$ streptomycin, in a humidified atmosphere with $5 \% \mathrm{CO}_{2}$ at $37^{\circ} \mathrm{C}$. Casticin (purity $\geq 98 \%$ ) was purchased from Chengdu Biopurify Phytochemicals Ltd. (Chengdu, China), as yellow crystals (molecular weight, 374.3 Da). It was dissolved in dimethyl-sulfoxide (DMSO) to prepare a $10 \mathrm{mmol} / \mathrm{l}$ stock solution, diluted in cell culture medium immediately before use. The following reagents were purchased from Hunan Clonetimes Biotech Co., Ltd. (Changsha, China): Antibodies against AMPKa (cat. no. 2532), p-AMPK (cat. no. 8324), ACC (cat. no. 9957), FoxO3a (cat. no. 2497), p-FoxO3a (cat. no. 9465), uPAR (cat. no. 9692) and CD133 (cat. no. 3570S) (Cell Signaling Technology, Inc., Danvers, MA. USA); antibodies targeting p-ACC (cat. no. sc-271965; Santa Cruz Biotechnology, Inc., Dallas, TX, USA) and human $\beta$-actin (cat. no. A4700; Sigma Chemicals; Merck KGaA, Darmstadt, Germany) were employed as well. Other reagents included Invitrogen ${ }^{\mathrm{TM}}$ Lipofectamine 2000 (Thermo Fisher Scientific, Inc., Waltham, MA, USA) and the growth supplements B-27 and N-2 (Invitrogen; Thermo Fisher Scientific, Inc.).

Sphere culture and self-renewal assay. To obtain spheres, the cells were cultured in stem cell-conditioned medium (DMEM/F12 medium supplemented with 0.02X B27, $20 \mathrm{ng} / \mathrm{ml}$ EGF, $20 \mathrm{ng} / \mathrm{ml} \mathrm{bFGF}, 0.4 \%$ BSA, $4 \mu \mathrm{g} / \mathrm{ml}$ insulin, $100 \mathrm{U} / \mathrm{ml}$ penicillin and $100 \mu \mathrm{g} / \mathrm{ml}$ streptomycin (Invitrogen; Thermo Fisher Scientific, Inc.) in ultra-low attachment 6-well plates (Corning Inc., Corning, NY, USA). When spheres reached $\geq 20$ cells, the suspension cultures were passaged every six days. Spheres were counted in 10 different high power fields using an inverted microscope (Nikon TS100; Nikon, Tokyo, Japan).

For future generation of spheres in vitro, sphere cells were collected by gentle centrifugation at $200 \mathrm{x} \mathrm{g}$ for $10 \mathrm{~min}$, dissociated into single-cell suspensions, and cultured to allow sphere regeneration.

To determine the sphere-formation rate, the dissociated cells or second-generation spheres treated with casticin (final concentrations of $1.0,3.0$ and $10.0 \mu \mathrm{mol} / 1$, respectively) were seeded at a density of 1,000 cells $/ \mathrm{ml}$ in 6 -well plates to generate new spheres. The total number of spheres was recorded after 6 days of culture. Sphere formation rate was calculated by dividing the total number of spheres formed by that of live cells seeded multiplied by 100 .

Clonogenic assay on soft agar. Each well of a 6-well culture plate was coated with $2 \mathrm{ml}$ bottom agar-medium mixture (DMEM, 10\% FBS, and 0.6\% agar). After solidification, $2 \mathrm{ml}$ top agar-medium mixture (DMEM, $10 \%$ FBS, and $0.3 \%$ Noble agar; BD Difco ${ }^{\mathrm{TM}}$; BD Biosciences, Franklin Lakes, NJ, USA) containing 1,000 cells treated as described above were added. After 14 days, the colonies formed ( $\geq 20$ cells) were counted under an inverted fluorescent microscope (Olympus CK40; Olympus Corp., Tokyo, Japan), with the representative views imaged. Colony formation rate was calculated by dividing the total number of colonies formed by that of live cells seeded multiplied by 100 .

Silencing by siRNA. Dissociated second-generation spheres were plated at $2.5 \times 10^{5}$ cells/well in 6 -well plates. After $24 \mathrm{~h}$, the siRNA-negative control (si-NC; Santa Cruz Biotechnology, Inc.) and AMPK- or FoxO3a-specific siRNAs (siAMPK or siFoxO3a; Shanghai GenePharma Co., Ltd., Shanghai, China) were transfected into cells, respectively, using Invitrogen ${ }^{\mathrm{TM}}$ Lipofectamine 2000 reagent (Thermo Fisher Scientific, Inc.) according to the manufacturer's instructions. Separate siRNAs were used for FoxO3A (5'-GACAAUAGCAACAAGUAU A-3') and AMPK (5'-GAGGAGCUAUUUGAUUA-3') (16). On-TARGET-plus control siRNAs (Thermo Fisher Scientific, Inc.) were used as control sequences.

Western blot analysis. Western blot analysis was carried out as described by Liu et al (17). Primary antibodies raised against AMPK $\alpha$, p-AMPK, FoxO3a, p-FoxO3a, uPAR, ALDH1, Bmi1, SOX2 and $\beta$-actin were used. Cells were lysed in lysis buffer for $20 \mathrm{~min}$ at $4^{\circ} \mathrm{C}$. Protein amounts were determined with the Bio-Rad assay system (Bio-Rad Laboratories, Hercules, CA, USA). Equal amounts (50 $\mu \mathrm{g})$ of total protein were fractionated by SDS-PAGE and transferred onto polyvinylidene fluoride membranes (PVDF) (Millipore, Billerica, MA, USA). Signals were detected using an ECL Advance Western blot analysis system (Amersham Pharmacia Biotech Inc., Piscataway, NJ, USA). Experiments were carried out in triplicate (ImageJ v.1.84; National Institutes of Health, Bethesda, MD, USA).

Immunohistochemical (IHC) analysis. Four micron-thick tissue sections were immunostained with uPAR-specific antibody. Immunostaining was performed using a Ventana 
Discovery Ultra (Ventana Medical Systems, Tucson, AZ, USA). Antigen retrieval was performed using $\mathrm{CC} 1$ for $40 \mathrm{~min}$ at $95^{\circ} \mathrm{C}$. IHC staining was followed by hematoxylin counterstaining. Slides were rinsed, dehydrated though alcohol and xylene and coverslipped.

In vivo tumorigenesis assessment. Twenty-four pathogen-free BALB/c-nu female mice (13-15 g) aged 4 weeks were purchased from the Animal Institute of the Chinese Academy of Medical Science (CAMS). All animal studies were performed in accordance with the standard protocols, and approved by the Ethics Committee of Hunan Normal University and the Committee of Experimental Animal Feeding and Management. Mice were randomly divided into 3 groups (4 mice/group), and maintained under standard conditions. Varying amounts of H446 cells $\left(10^{3}, 10^{4}\right.$ and $10^{5}$, respectively) were subcutaneously injected into 4-week-old female nude mice in the left flank; in parallel, second generation sphere-derived cells (LCSLCs, $10^{2}, 10^{3}$ and $10^{4}$, respectively) were subcutaneously injected into the right flank. Tumor growth was monitored visually every week, and the maximum tumor volume allowed was consistent with the IACUC guidelines (diameter, $1.5 \mathrm{~cm}$; area, $1.8 \mathrm{~cm}^{2}$; volume $\left.1.8 \mathrm{~cm}^{3}\right)$. Tumor volumes were calculated in accordance with the formula: $\mathrm{V}$ (transplanted tumor volume, $\mathrm{mm}^{3}$ ) $=\mathrm{L}$ (longest diameter, $\mathrm{mm}$ ) $\mathrm{x} \mathrm{W}$ (minimum diameter, $\mathrm{mm})^{2} \times 0.5$. After 8 weeks of tumor growth, the mice were euthanized using cervical vertebra luxation. The obtained tumor tissues were fixed in formalin and embedded in paraffin. Hematoxylin and eosin (H\&E) staining and immunohistochemical analysis were performed to assess tumor histology and tumor markers in the mouse xenografts.

Statistical analysis. SPSS 20.0 software (IBM Corp., Armonk, NY, USA) was used for analysis. Data are expressed as the mean \pm standard deviation (SD); $n=$ number of measurements. Multiple groups were compared by one-way analysis of variance (ANOVA); comparisons of group means were performed by the LSD method for normally distributed variables. Two-tailed t-test was also used as appropriate. $\mathrm{P}<0.05$ was considered statistically significant.

\section{Results}

Spheres from the H446 cell line show cancer stem-like cell characteristics. To evaluate the sphere-forming capabilities of the SCLC H446, H209 and H69 cell lines, sphere-formation rates of the three cell lines were assessed. The results showed that the sphere-forming capability of $\mathrm{H} 446$ cells was higher than that of both H209 and H69 cell lines (Fig. 1A). To determine the capability of H446, H209 and H69 cells for self-renewal initiation, these cells were submitted to several serial passages. As shown in Fig. 1B, sphere-formation rate was highest for second-generation spheres at the third sphere-formation process in the H446 cell line compared with other cell lines or spheres of another generation. Therefore, second-generation spheres of $\mathrm{H} 446$ cells were considered to be lung cancer stem-like cells (LCSLCs), and used for subsequent experiments.

We next compared the in vitro oncogenic capabilities of LCSLCs and H446 cells by sphere- and colony formation assays. The results showed that the size and population of tumor spheres from LCSLCs were larger than the values obtained for H446 cells (Fig. 1C). In addition, the colony formation ability was significantly enhanced in LCSLCs compared with H446 cells (Fig. 1D). These data suggested that LCSLCs had the capacity of self-renewal and oncogenic capabilities in vitro, and were highly enriched in second-generation spheres of H446 cells.

To further confirm the stem-like properties of LCSLCs, the protein expression levels of SCLC CSC-related markers were assessed in LCSLCs and H446 cells. Western blot analysis demonstrated that the protein expression levels of uPAR and CD133 were higher in the LCSLCs than these levels in the H446 cells (Fig. 1E).

To explore the role of AMPK/FoxO3a signaling in the in vitro carcinogenesis of H446-derived LCSLCs, the phosphorylated protein levels of AMPK, ACC and FoxO3a were determined. As shown in Fig. 1F, the phosphorylation levels of AMPK and ACC were lower, while FoxO3a protein phosphorylation was increased, in LCSLCs compared with H446 cells. These results suggest that inactivation of AMPK and FoxO3a were associated with carcinogenesis maintenance in vitro and the stem-like properties of H446-derived LCSLCs.

In addition, the abilities of LCSLCs and H446 cells to form tumors in BALB/c-nu mice were assessed. As many as $1 \times 10^{5}$ H446 cells were required to initiate stable tumor formation for 23-42 days after injection, while, as few as $1 \times 10^{3}$ LCSLCs were sufficient to generate visible tumors only 19-28 days post-injection (Fig. 1G). Furthermore, H\&E staining revealed similar histological characteristics in tumor xenografts derived from LCSLCs and H446 cells (Fig. 1H). Furthermore, uPAR protein amounts were higher in transplanted tumors of LCSLCs than those of H446 cells, as indicated by immunohistochemistry (Fig. 1H). These results provide sufficient evidence that $\mathrm{H} 446$ second-generation spheres possess LCSLC features such as increased in vitro carcinogenesis and in vivo tumorigenic potential.

Casticin inhibits in vitro carcinogenesis and activates AMPK/FoxO3a signaling in H446-derived LCSLCs. Similar to our previous findings (17), this study demonstrated that the indicated concentrations $(1.0,3.0$ and $10.0 \mu \mathrm{mol} / \mathrm{l})$ of casticin suppressed self-renewal capability by reducing the sphere-formation rate in H446-derived LCSLCs (Fig. 2A), in a dose-dependent manner. In addition, colony formation assay on soft agar showed that casticin significantly inhibited the colony forming ability of H446-derived LCSLCs (Fig. 2B), in a dose-dependent manner. To further confirm the inhibitory effects of casticin on CSC characteristics, we next assessed the expression levels of SCLC CSC bio-markers, including UPAR and CD133, in H446-derived LCSLCs treated with casticin at the indicated concentrations. The results showed that the protein levels of UPAR and CD133 were dose-dependently reduced by casticin (Fig. 2C). Taken together, these findings demonstrated that casticin could inhibit in vitro carcinogenesis and CSC characteristics in H446-derived LCSCs.

To test the hypothesis that casticin inhibits in vitro carcinogenesis and CSC characteristics in H446-derived LCSCs through activation of AMPK/FoxO3a signaling, the effects of casticin on the phosphorylated protein levels 
A

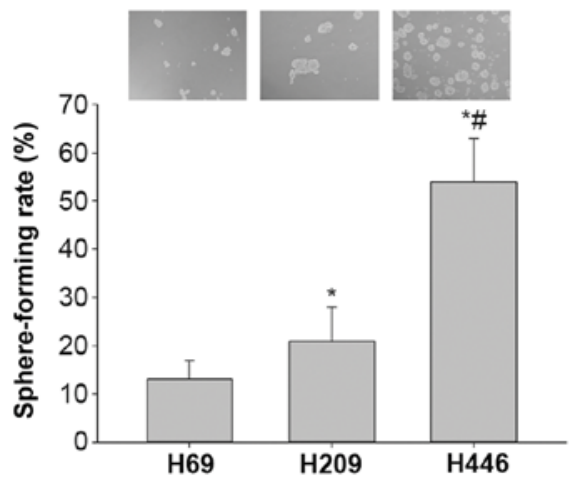

B

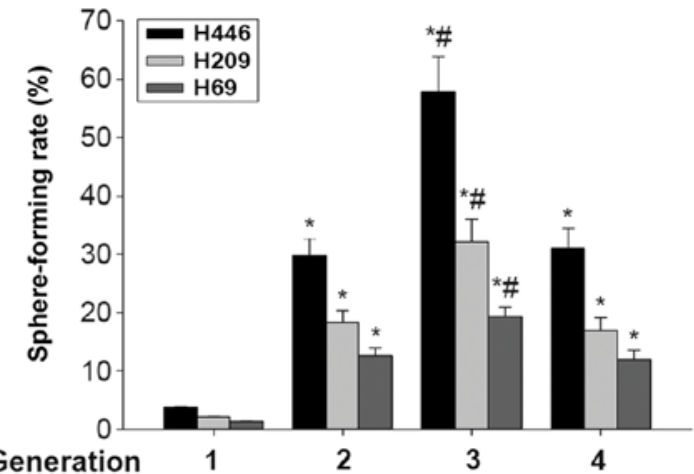

C
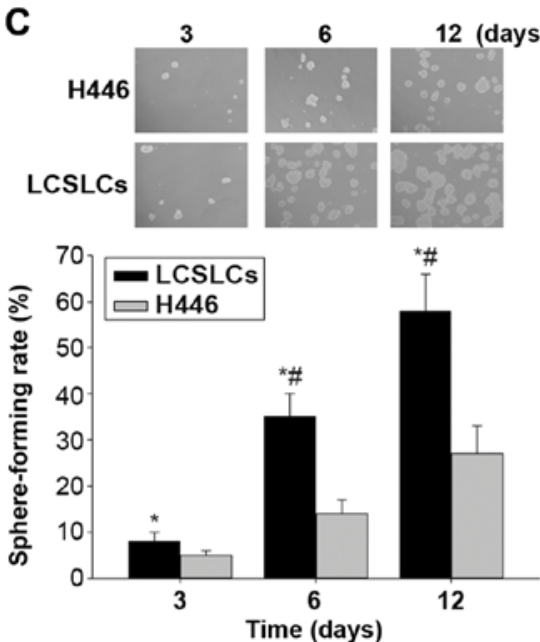

D

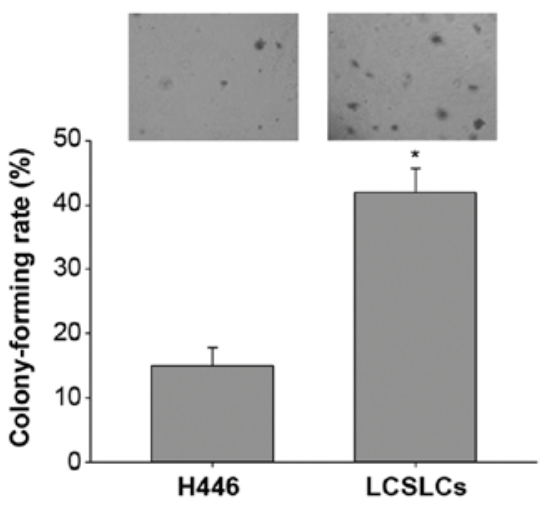

E
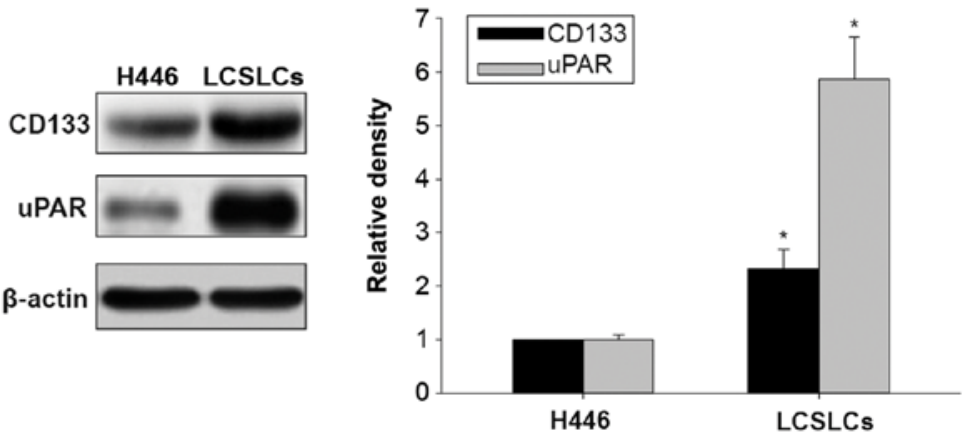

G

$\mathbf{F}$

\begin{tabular}{lccc}
\hline Cell type & $\begin{array}{c}\text { No. of inoculated } \\
\text { cells }\end{array}$ & $\begin{array}{c}\text { Tumor } \\
\text { incidence (n/4) }\end{array}$ & $\begin{array}{c}\text { Latency } \\
\text { (days) }\end{array}$ \\
\hline H446 & $1 \times 10^{3}$ & $0 / 4$ & - \\
& $1 \times 10^{4}$ & $0 / 4$ & - \\
& $1 \times 10^{5}$ & $4 / 4$ & $23 \sim 42$ \\
LCSLCs & $1 \times 10^{2}$ & $0 / 4$ & - \\
& $1 \times 10^{3}$ & $3 / 4$ & $19 \sim 28$ \\
& $1 \times 10^{4}$ & $4 / 4$ & $6 \sim 14$ \\
\hline
\end{tabular}

H
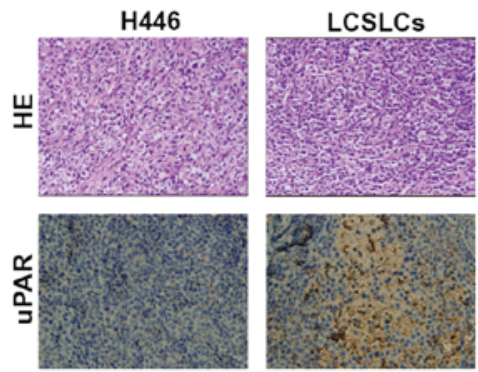

Figure 1. In vitro and in vivo carcinogenesis and AMPK/FoxO3a signaling activation in LCSLCs and H446 cells. (A) Representative micrographs of spheres obtained under a phase contrast microscope (magnification, x10, upper panel) and sphere-forming rates in $\mathrm{H} 69$, $\mathrm{H} 209$ and $\mathrm{H} 446 \mathrm{cells}$ (lower panel). $\mathrm{P}<0.05 \mathrm{vs}$. H69 cells. ${ }^{~} \mathrm{P}<0.05$ vs. H209 cells. (B) Sphere forming rates of $\mathrm{H} 69, \mathrm{H} 209$, and $\mathrm{H} 446$ in different generations. ${ }^{*} \mathrm{P}<0.05$ vs. first generation. ${ }^{\#} \mathrm{P}<0.05$ vs. second or fourth generation. (C) Representative micrographs of spheres obtained under a phase contrast microscope (magnification, $\mathrm{x} 10$, upper panel) and sphere-forming rates in LCSLCs and H446 cells (lower panel) at 3, 6 and 12 days, respectively. ${ }^{*} \mathrm{P}<0.05$ vs. H446 cells at the same time point. ${ }^{\#} \mathrm{P}<0.05$ vs. LCSLCs at 3 days. (D) Representative micrographs of colonies obtained under a phase contrast microscope (magnification, $\mathrm{x} 10$, upper panel) and colony-forming rates in LCSLCs and H446 cells (lower panel). (E) Representative western blot bands (left panel), and quantitative analysis of CD133 and uPAR protein expression levels in LCSLCs and H446 cells (right panel). (F) Representative western blot bands (left panel), and phosphorylation levels of AMPK $\alpha$, ACC and FoxO3a in LCSLCs and H446 cells (right panel). ${ }^{*} \mathrm{P}<0.05$ vs. H446 cells. (G) Ability of LCSLCs and H446 cells to form tumors in BALB/c-nu mice. (H) Hematoxylin and eosin (H\&E) staining and uPAR immunohistochemistry of tumor xenografts derived from LCSLCs and H446 cells. LCSLCs, lung cancer stem-like cells. 
A

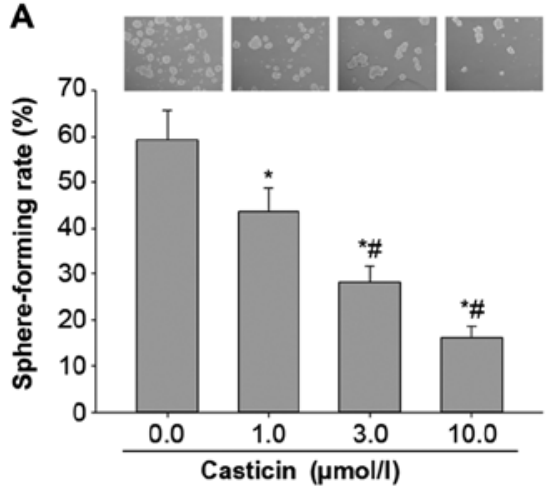

B

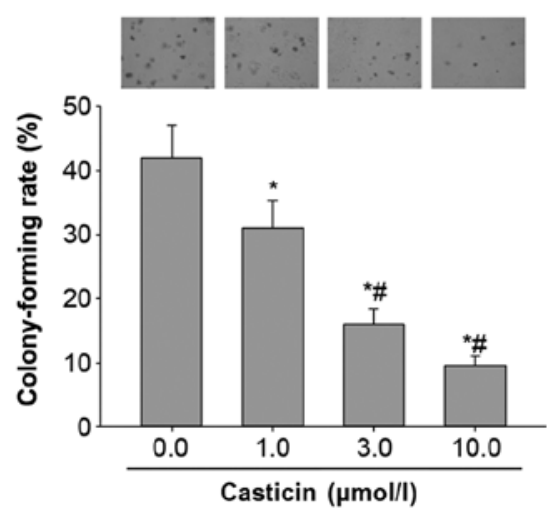

C

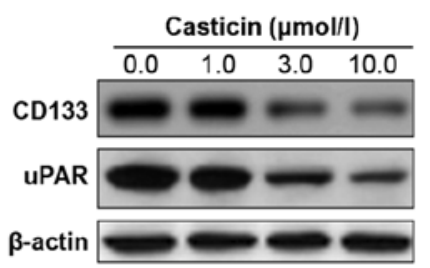

D
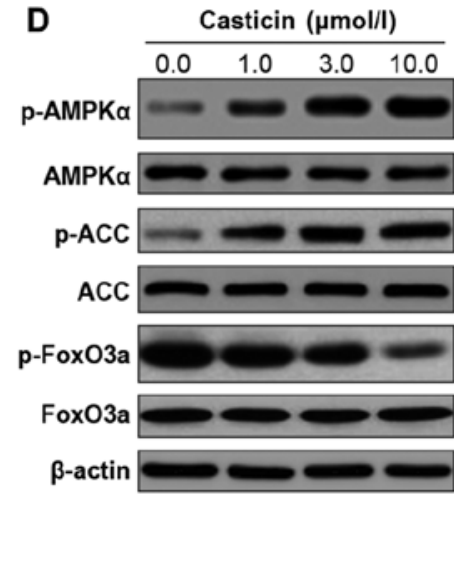
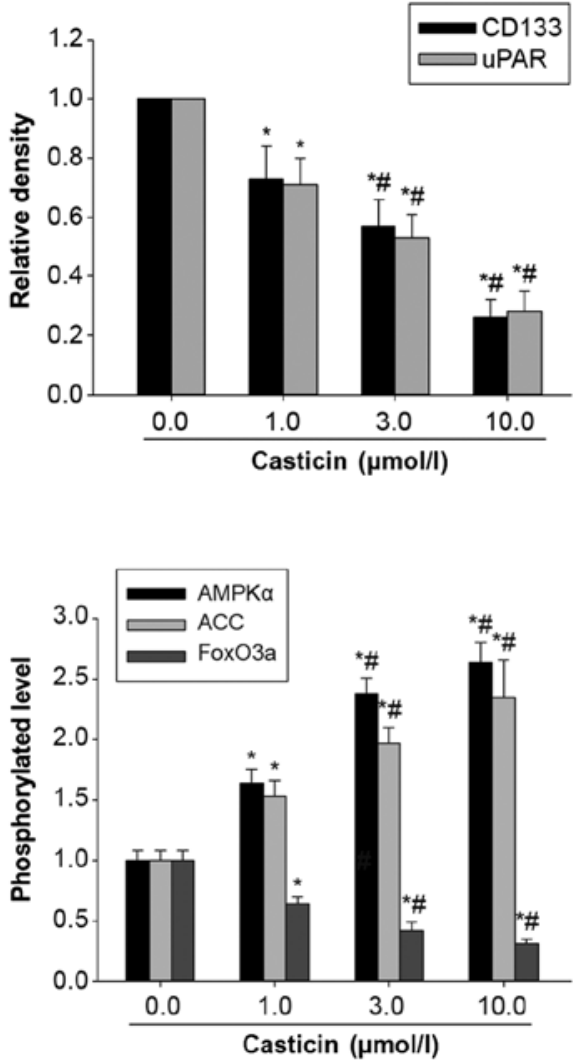

Figure 2. Effects of casticin on in vitro carcinogenesis and AMPK/FoxO3a signaling activation in H446-derived LCSLCs. (A) Representative micrographs of spheres obtained under a phase contrast microscope (magnification, x10, upper panel) and sphere-forming rates in H446-derived LCSLCs treated with casticin $(0.0,1.0,3.0$ and $10.0 \mu \mathrm{mol} / 1)$ (lower panel). (B) Representative micrographs of colonies obtained under a phase contrast microscope (magnification, $\mathrm{x} 10$, upper panel) and colony-forming rates in H446-derived LCSLCs treated with casticin (0.0, 1.0, 3.0 and 10.0 $\mu$ mol/1) (lower panel). (C) Representative western blot bands (left panel) and quantitative analysis of CD133 and uPAR protein expression levels in H446-derived LCSLCs treated with casticin (0.0, 1.0, 3.0 and $10.0 \mu \mathrm{mol} / \mathrm{l}$ ) (right panel). (D) Representative western blot bands (left) and phosphorylation levels of AMPK $\alpha$, ACC and FoxO3a in H446-derived LCSLCs treated with casticin $(0.0,1.0,3.0$ and $10.0 \mu \mathrm{mol} / 1)$ (right panel). ${ }^{*} \mathrm{P}<0.05$ vs. untreated group. ${ }^{\#} \mathrm{P}<0.05$ vs. LCSLCs treated with $1.0 \mu \mathrm{mol} / 1 \mathrm{casticin}$. LCSLCs, lung cancer stem-like cells.

of AMPK, ACC and FoxO3a were evaluated by western blot analysis. As shown in Fig. 2D, treatment with casticin resulted in significantly elevated phosphorylation levels of AMPK and ACC, and reduced FoxO3a phosphorylation, in a concentration-dependent manner. Collectively, these findings indicated that casticin inhibited in vitro carcinogenesis and CSC characteristics of H446-derived LCSCs, likely involving the activation of AMPK/FoxO3a signaling.

Effects of AMPK silencing on in vitro carcinogenesis and AMPK/FoxO3a signaling in H446-derived LCSLCs. AMPK is a central cellular energetic biosensor that regulates a broad array of cellular metabolic routes activated by nutrient deprivation, mitochondrial dysfunction, oxidative stress and cytokines (18). Previous studies have demonstrated that casticin induces apoptosis through reactive oxygen species-mediated mitochondrial signaling pathways in cervical (19) and lung (20) cancers. Therefore, we hypothesized that the inhibitory effects of casticin on H446-derived LCSLCs may also involve AMPK activation. To test this, we firstly knocked down AMPK with siRNA in H446-derived LCSLCs, and evaluated the phosphorylated protein levels of AMPK, ACC and FoxO3a. As shown in Fig. 3A, transfection with AMPK siRNA resulted in reduced AMPK protein expression, decreased ACC phosphorylation, and increased phosphorylation levels of FoxO3a, compared with the untreated or control siRNA-treated H446-derived LCSLCs. Moreover, AMPK knockdown enhanced sphere and colony formation abilities, compared with the untreated or control siRNA-treated H446-derived LCSLCs (Fig. 3B and C). Accordingly, the protein expression levels of uPAR and CD133 were increased in the H446-derived LCSLCs transfected with AMPK siRNA compared with the untreated or control siRNA-treated counterparts (Fig. 3D). These results suggested that AMPK knockdown blocked AMPK/FoxO3a signaling, and enhanced in vitro carcinogenesis and CSC characteristics in H446-derived LCSLCs.

Effects of casticin on in vitro carcinogenesis and AMPK/FoxO3a signaling activation in H446-derived LCSLCs transfected with AMPK siRNA. To assess whether the inhibitory effects of casticin on oncogenicity in H446-derived LCSLCs is affected by AMPK regulation, casticin (0 or $3.0 \mu \mathrm{mol} / \mathrm{l}$ ) was administered to H446-derived LCSLCs transfected with AMPK- and control siRNAs, respectively. Compared with the control siRNA group, AMPK silencing not only reduced AMPK levels and ACC phosphorylation, but also antagonized elevated phosphorylation levels of AMPK 

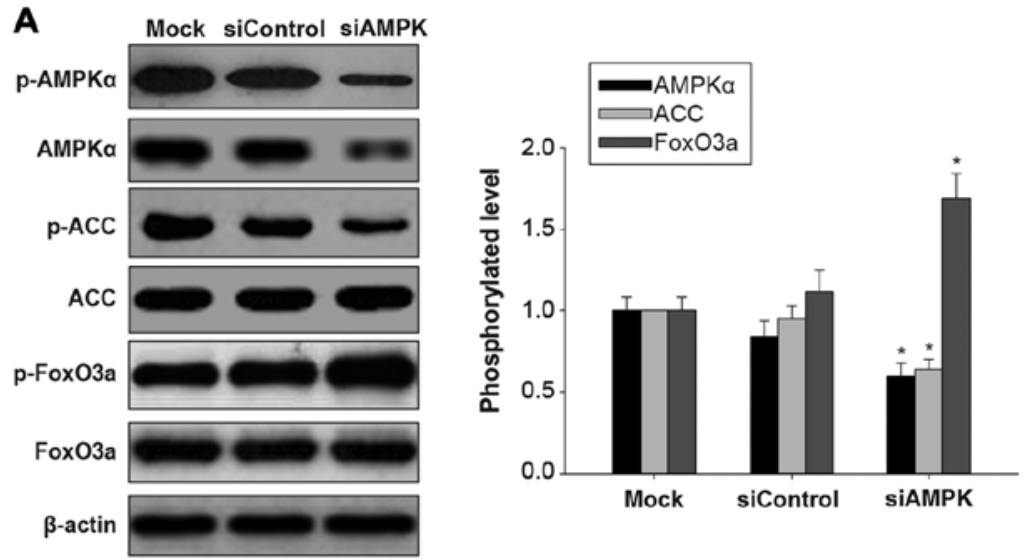

B

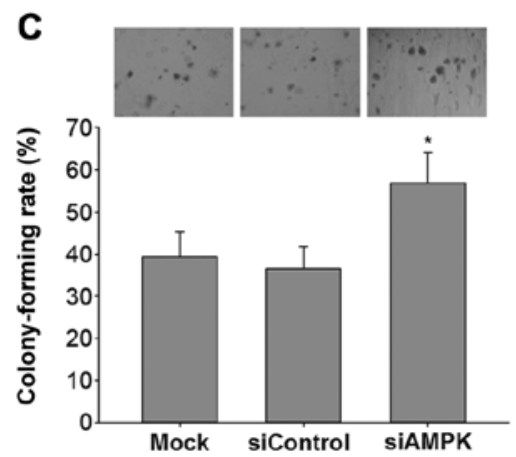

D
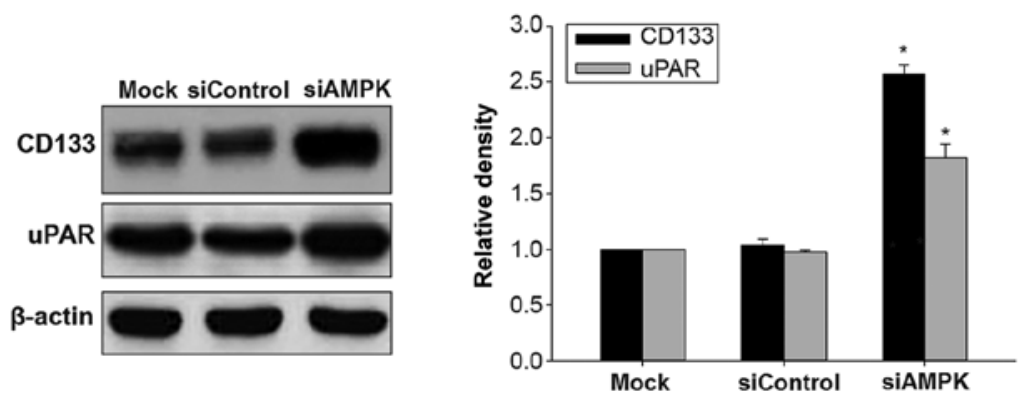

Figure 3. Effects of AMPK siRNA on in vitro carcinogenesis and AMPK/FoxO3a signaling in H446-derived LCSLCs. (A) Representative western blot bands (left panel) and the phosphorylation levels of AMPKa, ACC, and FoxO3a in H446-derived LCSLCs transfected with AMPK siRNA (siAMPK) and untreated (Mock) cells or H446-derived LCSLCs transfected with control siRNA (siControl) (right panel). (B) Representative micrographs of spheres obtained under a phase contrast microscope (magnification, x10, upper panel) and sphere-forming rates in H446-derived LCSLCs transfected with AMPK siRNA (siAMPK) and untreated (Mock) cells or H446-derived LCSLCs transfected with control siRNA (siControl) (lower panel). (C) Representative micrographs of colonies obtained under a phase contrast microscope (magnification, x10, upper panel) and colony-forming rates in H446-derived LCSLCs transfected with AMPK siRNA (siAMPK) and untreated (Mock) cells or H446-derived LCSLCs transfected with control siRNA (siControl) (lower panel). (D) Representative western blot bands (left) and quantitative analysis of CD133 and uPAR protein expression levels in H446-derived LCSLCs transfected with AMPK siRNA (siAMPK) and untreated (Mock) cells or H446-derived LCSLCs transfected with control siRNA (siControl) (right panel). ${ }^{*} \mathrm{P}<0.05$ vs. Mock or siControl. LCSLCs, lung cancer stem-like cells.

and ACC in response to casticin administration $(3.0 \mu \mathrm{mol} / \mathrm{l})$ in H446-derived LCSLCs (Fig. 4A). In addition, AMPK knockdown also elevated the phosphorylation levels of FoxO3a, while attenuating the reduction of FoxO3a phosphorylation after treatment with casticin $(3.0 \mu \mathrm{mol} / \mathrm{l})$ in H446-derived LCSLCs (Fig. 4A). Furthermore, AMPK knockdown not only enhanced self-renewal and colony formation capabilities, but also counteracted the inhibitory effects of casticin on carcinogenesis in H446-derived LCSLCs in vitro (Fig. 4B and C). Meanwhile, transfection with AMPK siRNA resulted in increased UPAR and CD133 protein levels, reversing the inhibitory effects of casticin on uPAR and CD133 expression in H446-derived LCSLCs (Fig. 4D). These results suggested that AMPK activation may be upstream of FoxO3a activation in response to casticin stimulation in H446-derived LCSLCs.

Effects of FoxO3a silencing on in vitro carcinogenesis and AMPK/FoxO3a signaling activation in H446-derived LCSLCs. FoxO3a, a downstream effector of AMPK, is associated with a variety of cell processes, including cell cycle progression, apoptosis, stress, detoxification, DNA repair, glucose metabolism and differentiation (21). To assess whether the in vitro effects of casticin on carcinogenesis and CSC characteristics in H446-derived LCSLCs involve AMPK/FoxO3a signaling, FoxO3a was silenced. As shown in Fig. 5A, FoxO3a knockdown mainly resulted in decreased FoxO3a protein levels, with no effects on AMPK and ACC levels or phosphorylation. Furthermore, FoxO3a knockdown increased sphere and colony formation rates (Fig. 5B and C) as well as uPAR and CD133 protein levels (Fig. 5D) in H446-derived LCSLCs. These results indicated that activation of FoxO3a could inhibit in vitro carcinogenesis and CSC characteristics in H446-derived LCSLCs, and FoxO3a may be a downstream target of AMPK.

Effects of casticin on in vitro carcinogenesis and AMPK/FoxO3a signaling activation in H446-derived LCSLCs transfected with FoxO3a siRNA. To further assess whether the effects of casticin on in vitro carcinogenesis and CSC characteristics are mediated by AMPK/FoxO3a signaling, casticin ( 0 or $3.0 \mu \mathrm{mol} / \mathrm{l})$ was administered to H446-derived LCSLCs transfected with FoxO3a or control siRNA. As expected, FoxO3a knockdown synergistically reduced FoxO3a protein phosphorylation, with slight effects on elevated AMPK and ACC phosphorylation levels associated with casticin (3.0 $\mu \mathrm{mol} / \mathrm{l})$ in H446-derived LCSLCs (Fig. 6A). 
A

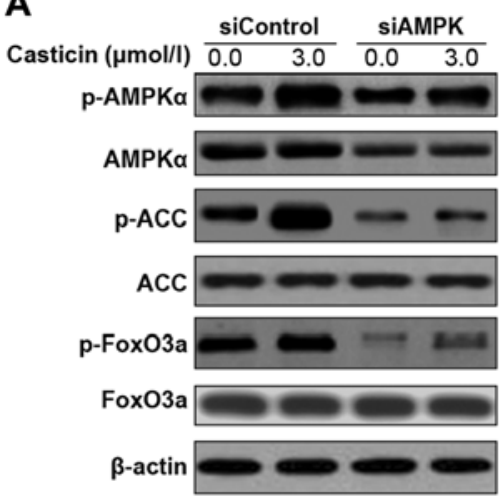

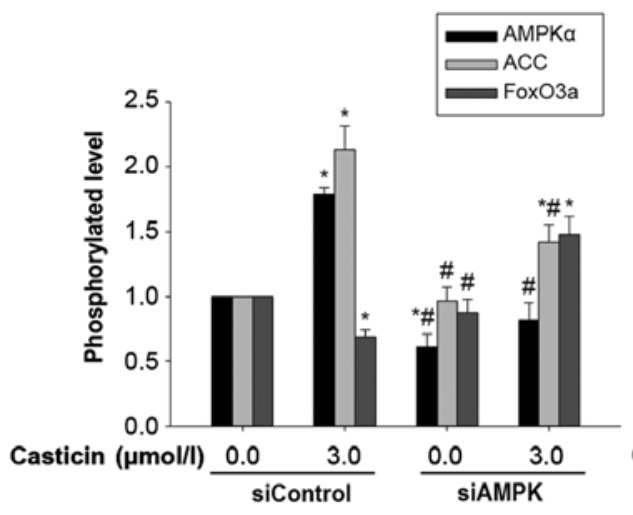

D
B

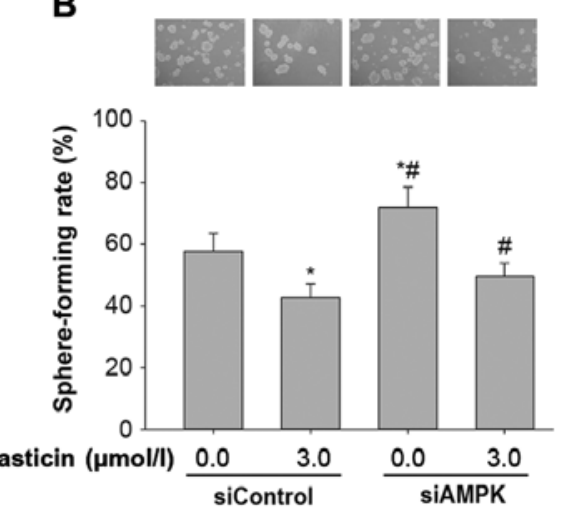

C

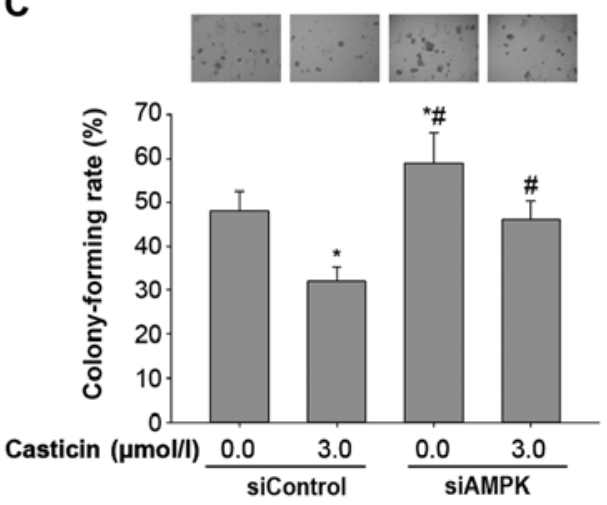

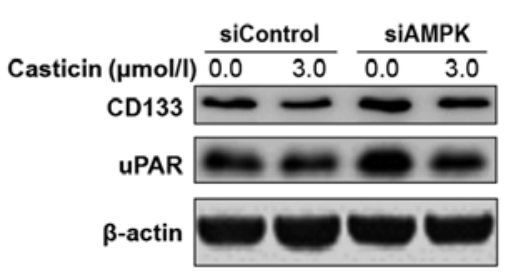
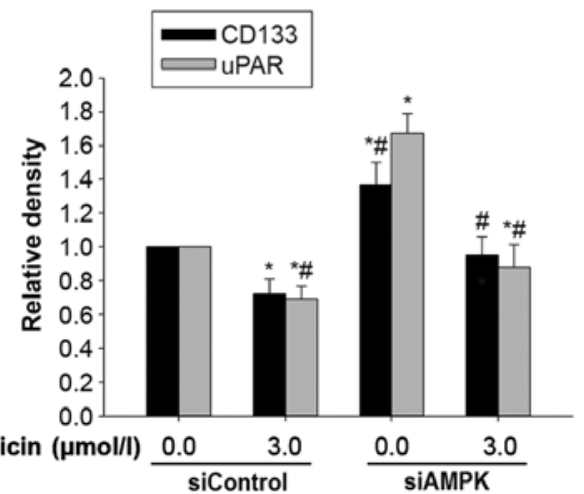

Figure 4. Effects of casticin on in vitro carcinogenesis and AMPK/FoxO3a signaling in H446-derived LCSLCs transfected with AMPK siRNA. (A) Representative western blot bands (left panel) and phosphorylation levels of AMPK $\alpha$, ACC and FoxO3a in H446-derived LCSLCs transfected with control siRNA (siControl) or AMPK siRNA (siAMPK) in the absence or presence of casticin (3.0 $\mu$ mol/l) (right panel). (B) Representative micrographs of spheres obtained under a phase contrast microscope (magnification, x10, upper panel) and sphere-forming rates in H446-derived LCSLCs transfected with control (siControl) or AMPK siRNA (siAMPK) in the absence or presence of casticin (3.0 $\mu \mathrm{mol} / \mathrm{l})$ (right panel). (C) Representative micrographs of colonies obtained under a phase contrast microscope (magnification, x10, upper panel) and colony-forming rates in H446-derived LCSLCs transfected with control (siControl) or AMPK siRNA (siAMPK) in the absence or presence of casticin (3.0 $\mu \mathrm{mol} / \mathrm{l})$ (right panel). (D) Representative western blot bands (left panel) and quantitative analysis of CD133 and uPAR protein expression levels in H446-derived LCSLCs transfected with control (siControl) or AMPK siRNA (siAMPK) in the absence or presence of casticin $(3.0 \mu \mathrm{mol} / \mathrm{l})$ (right panel). ${ }^{*} \mathrm{P}<0.05 \mathrm{vs}$. siControl. ${ }^{\#} \mathrm{P}<0.05 \mathrm{vs}$. siControl in response to casticin (3.0 $\mu$ mol/1). LCSLCs, lung cancer stem-like cells.

As shown in Fig. 6B and C, FoxO3a silencing neutralized the inhibitory effects of casticin $(3.0 \mu \mathrm{mol} / \mathrm{l})$ on sphere and colony formation abilities in H446-derived LCSLCs. Consistently, FoxO3a knockdown weakened the decreased expression levels of UPAR and CD133 observed in H446-derived LCSLCs in response to treatment with casticin $(3.0 \mu \mathrm{mol} / \mathrm{l})$ (Fig. 6D). These findings clearly indicated that the inhibitory effects of casticin on in vitro carcinogenesis and CSC properties were mediated by AMPK/FoxO3a signaling in H446-derived LCSLCs.

\section{Discussion}

The present study confirmed that casticin reduced the in vitro carcinogenesis and cancer stem cell (CSC) characteristics, and the molecular mechanism involved activation of AMPK/FoxO3 signaling in H446-derived lung cancer stem-like cells (LCSLCs). CSCs are a small population of tumor cells with the properties of self-renewal, multi-differentiation potential, and high aggressiveness and tumorigenesis in vivo (4-6). This study firstly examined second-generation spheres from the H446 cell line, namely, H446-derived LCSLCs, using sphere- and colony-formation assays, western blot analysis and xenografts in nude mice. The H446-derived LCSLCs were characterized by stronger sphere- and colony-forming capacities and overexpression of SCLC CSC biomarkers, including UPAR and CD133, compared with H446 cells. In addition, xenograft assays in nude mice provided important evidence that H446-derived LCSLCs had higher capability of tumorigenesis in vivo compared with H466 cells. Therefore, second-generation spheres from the H446 cell line could be considered as LCSLCs.

He et al (22) found that casticin inhibited epithelialmesenchymal transition (EMT) in liver cancer stem cells from the SMMC-7721 cell line by downregulating Twist. He et al (23) demonstrated casticin also suppressed self-renewal ability in liver cancer stem cells from the MHCC97 cell line. Our previous study showed that casticin suppressed the self-renewal and invasion abilities of LCSLCs from NSCLC A549 cells through p-Akt downregulation (17). The present study demonstrated that casticin efficaciously reduced sphere and colony formation abilities, and downregulated SCLC CSC biomarkers such as UPAR and CD133, in H446-derived LCSLCs. These results clearly indicated that casticin reduced 

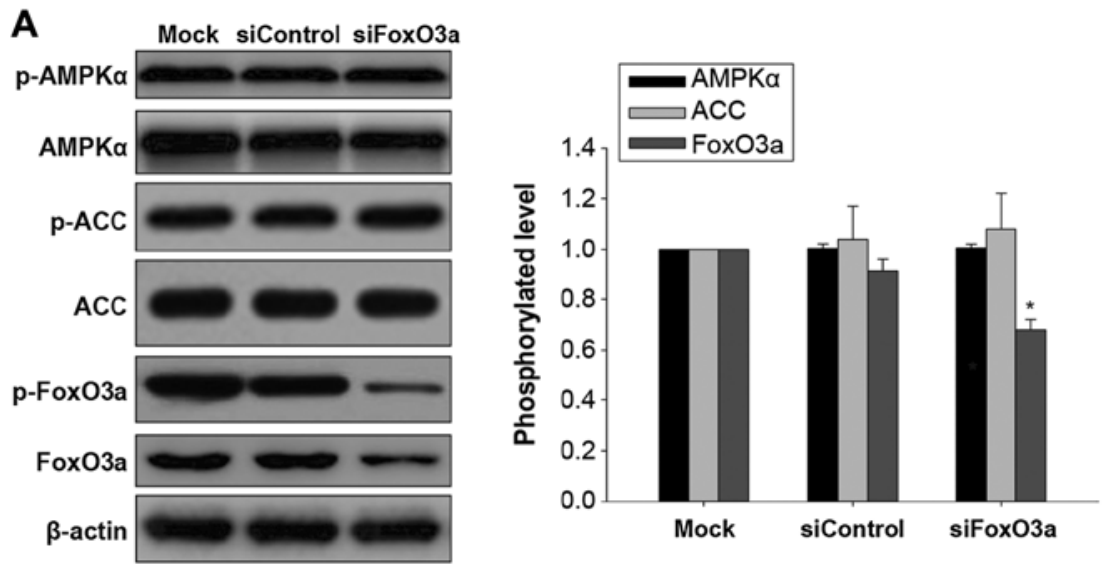

B
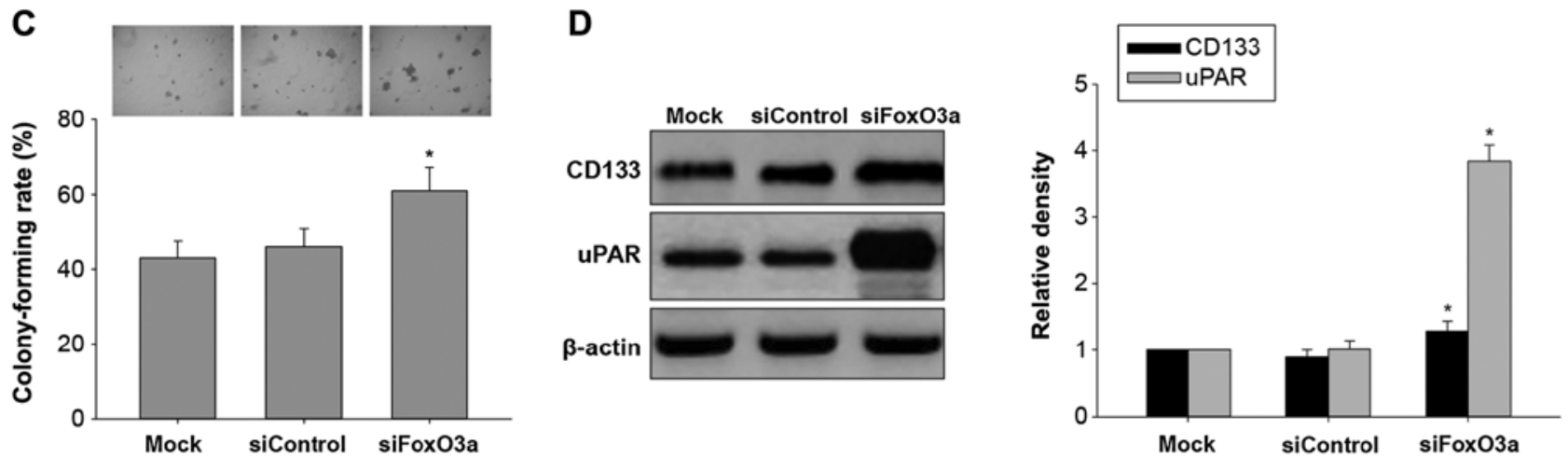

Figure 5. Effects of FoxO3a silencing on in vitro carcinogenesis and AMPK/FoxO3a signaling in H446-derived LCSLCs. (A) Representative western blot bands (left panel) and phosphorylation levels of AMPK $\alpha$, ACC, and FoxO3a in H446-derived LCSLCs transfected with FoxO3a siRNA (siFoxO3a) and untreated (Mock) cells or H446-derived LCSLCs transfected with control siRNA (siControl) (right panel). (B) Representative micrographs of spheres obtained under a phase contrast microscope (magnification, x10, upper panel) and sphere-forming rates in H446-derived LCSLCs transfected with FoxO3a siRNA (siFoxO3a) and untreated (Mock) cells or H446-derived LCSLCs transfected with control siRNA (siControl) (lower panel). (C) Representative micrographs of colonies obtained under a phase contrast microscope (magnification, x10, upper panel) and colony-forming rates in H446-derived LCSLCs transfected with FoxO3a siRNA (siFoxO3a) and untreated (Mock) cells or H446-derived LCSLCs transfected with control siRNA (siControl) (lower panel). (D) Representative western blot bands (left panel) and quantitative analysis of CD133 and uPAR protein expression levels in H446-derived LCSLCs transfected with FoxO3a siRNA (siFoxO3a) and untreated (Mock) cells or H446-derived LCSLCs transfected with control siRNA (siControl) (right panel). "P<0.05 vs. Mock or siControl. LCSLCs, lung cancer stem-like cells.

in vitro carcinogenesis and CSC characteristics in SCLC LCSLCs. However, the molecular mechanism by which casticin inhibits SCLC LCSLCs remained poorly understood.

Given that casticin suppresses cell growth in ovarian cancer (SKOV3) cells through FOXO3a activation (13), we aimed to ascertain whether casticin inhibits in vitro carcinogenesis and CSC characteristics in SCLC LCSLCs by activating FOXO3a, a transcription factor inactivated by AMPK $(14-16,24)$. We first found that casticin significantly increased the phosphorylation levels of AMPK and ACC, in a concentration-dependent manner. These findings indicated that casticin increased AMPK activity. More importantly, AMPK knockdown not only reduced AMPK expression and ACC phosphorylation levels, but also antagonized the elevated phosphorylation levels of AMPK and ACC in response to casticin administration in H446-derived LCSLCs. Therefore, these findings suggest that AMPK activation may be required for response to casticin stimulation in H446-derived LCSLCs.

It was reported that AMPK acts as an upstream regulator of Foxo3a $(8-10,14-16,24,25)$. This study provided several lines of evidence that casticin activates Foxo3a in an AMPK-dependent manner. Indeed, AMPK knockdown resulted in elevated phosphorylation levels of FoxO3a, and attenuated the decreased FoxO3a phosphorylation associated with casticin administration in H446-derived LCSLCs. In addition, FoxO3a silencing mainly led to decreased FoxO3a protein levels, with no effects on AMPK and ACC expression and phosphorylation associated with casticin administration. Mechanistically, casticin had no effect on AMPK protein expression, but suppressed AMPK-mediated FoxO3a phosphorylation. Functionally, FoxO3a knockdown mimicked the effects AMPK knockdown on sphere- and colony-forming capabilities, as well as on protein expression levels of the CSC markers uPAR and CD133, in H446-derived LCSLCs. These findings suggest that the inhibitory effects of casticin on in vitro carcinogenesis and CSC properties may be mediated by activation of AMPK/FoxO3a signaling in H446-derived LCSLCs.

Overexpression of UPAR is strongly correlated with the malignant cancer phenotype and poor prognosis $(26,27)$. In breast cancer, elevated uPAR expression is an independent prognostic marker of reduced relapse-free survival (27). Consistently, high uPAR levels in primary tumors indicate aggressive gastric cancer (28). LeBeau et al and others 
A

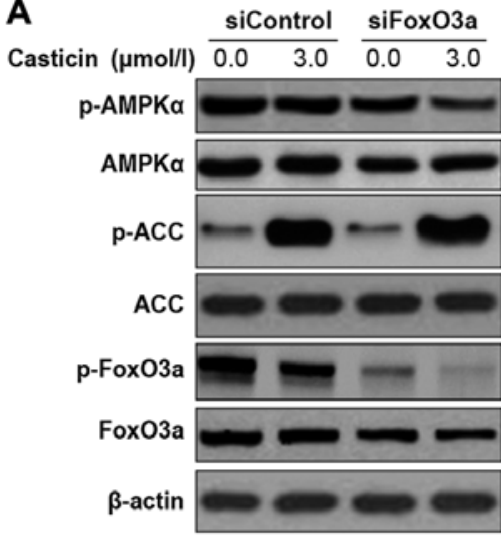

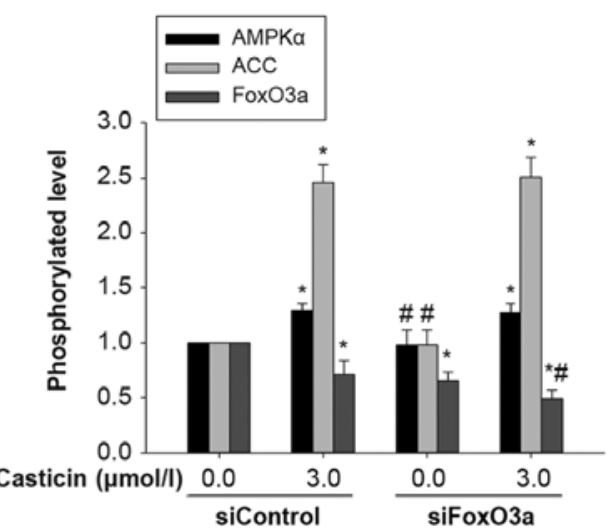

D
B

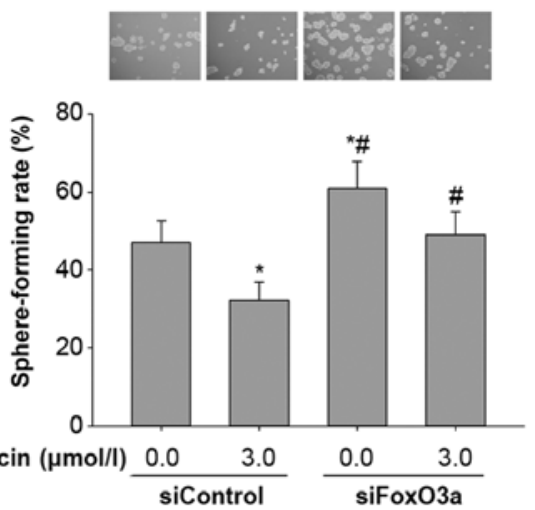

C

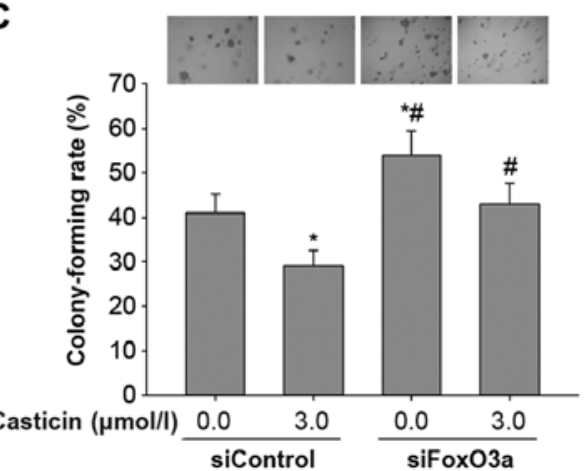

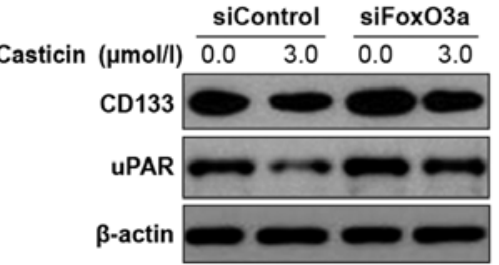

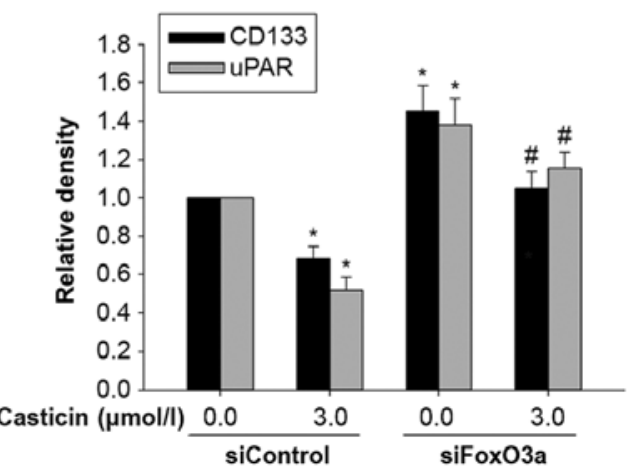

Figure 6. Effects of casticin on in vitro carcinogenesis and AMPK/FoxO3a signaling in H446-derived LCSLCs transfected with FoxO3a siRNA. (A) Representative western blot bands (left panel) and phosphorylation levels of AMPK $\alpha$, ACC and FoxO3a in H446-derived LCSLCs transfected with control (siControl) or FoxO3a siRNA (siFoxO3a) in the absence or presence of casticin $(3.0 \mu \mathrm{mol} / \mathrm{l})$ (right panel). (B) Representative micrographs of spheres obtained under a phase contrast microscope (magnification, x10, upper panel) and sphere-forming rates in H446-derived LCSLCs transfected with control (siControl) or FoxO3a siRNA (siFoxO3a) in the absence or presence of casticin (3.0 $\mu \mathrm{mol} / \mathrm{l})$ (lower panel). (C) Representative micrographs of colonies obtained under a phase contrast microscope (magnification, x10, upper panel) and colony-forming rates in H446-derived LCSLCs transfected with control (siControl) or FoxO3a siRNA (siFoxO3a) in the absence or presence of casticin (3.0 $\mu \mathrm{mol} / \mathrm{l})$ (lower panel). (D) Representative western blot band (left panel) and quantitative analysis of CD133 and uPAR protein expression levels in H446-derived LCSLCs transfected with control (siControl) or FoxO3a siRNA (siFoxO3a) in the absence or presence of casticin $(3.0 \mu \mathrm{mol} / \mathrm{l})$ (right panel) ${ }^{*} \mathrm{P}<0.05 \mathrm{vs}$. siControl. ${ }^{~} \mathrm{P}<0.05 \mathrm{vs}$. siControl in response to casticin $(3.0 \mu \mathrm{mol} / \mathrm{l})$. LCSLCs, lung cancer stem-like cells.

demonstrated that a stem cell-like cell population may be enriched in spheres expressing the UPAR cell surface marker in H446 cells $(27,29)$. Furthermore, inhibition of uPAR results in prominently increased $\mathrm{p} 27^{\mathrm{Kip} 1}$ expression, which correlates with decreased p-PI3K and p-AKT levels, as well as increased FOXO3a protein amounts $(30,31)$.The current findings suggest that the inhibitory effects of casticin on in vitro carcinogenesis and CSC properties involve UPAR downregulation. However, the interaction of FoxO3a with uPAR warrants further investigation.

In summary, this study firstly provided evidence that AMPK plays a role in regulating in vitro carcinogenesis and CSC properties by activating FoxO3a in H446-derived LCSLCs. In addition, the above findings indicated that the inhibitory effects of casticin on in vitro carcinogenesis and CSC properties in H446-derived LCSLCs, including sphere and colony formation abilities, and increased levels of CSC biomarkers (e.g. uPAR and CD133), are associated with activation of AMPK/FoxO3a signaling. Overall, this study raises the novel and intriguing idea that interventions to modulate AMPK, a known cellular metabolic sensor by casticin, could contribute to preventing and treating human SCLC by targeting LCSLCs.

\section{Acknowledgements}

Not applicable.

\section{Funding}

The present study was supported by the Project of NSFC (nos. 30760248 and 81172375), the Project of Scientific Research of Hunan Province, granted by the Health and Family Planning Commission of Hunan Province (no. B2013-098).

\section{Availability of data and materials}

The datasets used during the present study are available from the corresponding author upon reasonable request.

\section{Authors' contributions}

Professor JC conceived and designed the study. QG, XC and XY performed the experiments. QG and XC wrote the manuscript. Professor JC and WZ reviewed and edited the manuscript. Professor WZ was also involved in the conception of the study. All authors read and approved the manuscript 
and agree to be accountable for all aspects of the research in ensuring that the accuracy or integrity of any part of the work are appropriately investigated and resolved.

\section{Ethics approval and consent to participate}

All animal studies were performed in accordance with the standard protocols, and approved by the Ethics Committee of Hunan Normal University and the Committee of Experimental Animal Feeding and Management.

\section{Patient consent for publication}

Not applicable.

\section{Competing interests}

The authors declare that they have no conflict of interest.

\section{References}

1. DeSantis CE, Lin CC, Mariotto AB, Siegel RL, Stein KD, Kramer JL, Alteri R, Robbins AS and Jemal A: Cancer treatment and survivorship statistics, 2014. CA Cancer J Clin 64: 252-271, 2014.

2. Zakaria N, Satar NA, Abu Halim NH, Ngalim SH, Yusoff NM, Lin J and Yahaya BH: Targeting lung cancer stem cells: Research and clinical impacts. Front Oncol 7: 80, 2017.

3. Zhang Z, Zhou Y, Qian H, Shao G, Lu X, Chen Q, Sun X, Chen D, Yin R, Zhu H, et al: Stemness and inducing differentiation of small cell lung cancer NCI-H446 cells. Cell Death Dis 4: e633, 2013.

4. Rahman M, Deleyrolle L, Vedam-Mai V, Azari H, Abd-El-Barr M and Reynolds BA: The cancer stem cell hypothesis: Failures and pitfalls. Neurosurgery 68: 531-545, 2011.

5. Tysnes BB and Bjerkvig R: Cancer initiation and progression: Involvement of stem cells and the microenvironment. Biochim Biophys Acta 1775: 283-297, 2007.

6. Qiu X, Wang Z, Li Y, Miao Y, Ren Y and Luan Y: Characterization of sphere-forming cells with stem-like properties from the small cell lung cancer cell line H446. Cancer Lett 323: 161-170, 2012.

7. Xiao J, Mu JS, Liu TR and Xu HN: Dig the root of cancer: Targeting cancer stem cells therapy. J Med Discov 2: 1-6, 2017.

8. Sato A, Sunayama J, Okada M, Watanabe E, Seino S, Shibuya K, Suzuki K, Narita Y, Shibui S, Kayama T, et al: Glioma-initiating cell elimination by metformin activation of FOXO3 via AMPK. Stem Cells Transl Med 1: 811-824, 2012.

9. Yung MM, Chan DW, Liu VW, Yao KM and Ngan HY: Activation of AMPK inhibits cervical cancer cell growth through AKT/FOXO3a/FOXM1 signaling cascade. BMC Cancer 13: 327, 2013.

10. Queiroz EA, Puukila S, Eichler R, Sampaio SC, Forsyth HL, Lees SJ, Barbosa AM, Dekker RF, Fortes ZB and Khaper N: Metformin induces apoptosis and cell cycle arrest mediated by oxidative stress, AMPK and FOXO3a in MCF-7 breast cancer cells. PLoS One 9: e98207, 2014.

11. He L, Yang X, Cao X, Liu F, Quan M and Cao J: Casticin induces growth suppression and cell cycle arrest through activation of FOXO3a in hepatocellular carcinoma. Oncol Rep 29: 103-108, 2013.

12. Liu LP, Cao XC, Liu F, Quan MF, Sheng XF and Ren KQ: Casticin induces breast cancer cell apoptosis by inhibiting the expression of forkhead box protein M1. Oncol Lett 7: 1711-1717, 2014.

13. Jiang L, Cao XC, Cao JG, Liu F, Quan MF, Sheng XF and Ren KQ: Casticin induces ovarian cancer cell apoptosis by repressing FoxM1 through the activation of FOXO3a. Oncol Lett 5: 1605-1610, 2013.
14. Shrestha A, Nepal S, Kim MJ, Chang JH, Kim SH, Jeong GS, Jeong CH, Park GH, Jung S, Lim J, et al: Critical role of AMPK/FoxO3A axis in globular adiponectin-induced cell cycle arrest and apoptosis in cancer cells. J Cell Physiol 231: 357-369, 2016.

15. Zhao Y, Sun Y, Ding Y, Wang X, Zhou Y, Li W, Huang S, Li Z, Kong L, Guo Q, et al: GL-V9, a new synthetic flavonoid derivative, ameliorates DSS-induced colitis against oxidative stress by up-regulating Trx-1 expression via activation of AMPK/FOXO3a pathway. Oncotarget 6: 26291-26307, 2015.

16. Zheng F, Wu J, Zhao S, Luo Q, Tang Q, Yang L, Li L, Wu W and Hann SS: Baicalein increases the expression and reciprocal interplay of RUNX3 and FOXO3a through crosstalk of AMPKalpha and MEK/ERK1/2 signaling pathways in human non-small cell lung cancer cells. J Exp Clin Cancer Res 34: 41, 2015.

17. Liu F, Cao X, Liu Z, Guo H, Ren K, Quan M, Zhou Y, Xiang H and Cao J: Casticin suppresses self-renewal and invasion of lung cancer stem-like cells from A549 cells through down-regulation of pAkt. Acta Biochim Biophys Sin 46: 15-21, 2014.

18. Bonini MG and Gantner BN: The multifaceted activities of AMPK in tumor progression-why the 'one size fits all' definition does not fit at all? IUBMB Life 65: 889-896, 2013.

19. Chen D, Cao J, Tian L, Liu F and Sheng X: Induction of apoptosis by casticin in cervical cancer cells through reactive oxygen species-mediated mitochondrial signaling pathways. Oncol Rep 26: 1287-1294, 2011.

20. Zhou Y, Peng Y, Mao QQ, Li X, Chen MW, Su J, Tian L, Mao NQ, Long LZ, Quan MF, et al: Casticin induces caspase-mediated apoptosis via activation of mitochondrial pathway and upregulation of DR5 in human lung cancer cells. Asian Pac J Trop Med 6: 372-378, 2013.

21. Myatt SS and Lam EW: The emerging roles of forkhead box (Fox) proteins in cancer. Nat Rev Cancer 7: 847-859, 2007.

22. He M, Cao XC, He GC, Sheng XF, Ai XH and Wu YH: Casticin inhibits epithelial-mesenchymal transition of liver cancer stem cells of the SMMC-7721 cell line through downregulating Twist. Oncol Lett 7: 1625-1631, 2014.

23. He G, Cao X, He M, Sheng X, Wu Y and Ai X: Casticin inhibits self-renewal of liver cancer stem cells from the MHCC97 cell line. Oncol Lett 7: 2023-2028, 2014.

24. Chou CC, Lee KH, Lai IL, Wang D, Mo X, Kulp SK, Shapiro CL and Chen CS: AMPK reverses the mesenchymal phenotype of cancer cells by targeting the Akt-MDM2-Foxo3a signaling axis. Cancer Res 74: 4783-4795, 2014.

25. Guo Q, Liu Z, Jiang L, Liu M, Ma J, Yang C, Han L, Nan K and Liang $X$ : Metformin inhibits growth of human non-small cell lung cancer cells via liver kinase B-1-independent activation of adenosine monophosphate-activated protein kinase. Mol Med Rep 13: 2590-2596, 2016.

26. Gutova M, Najbauer J, Gevorgyan A, Metz MZ, Weng Y, Shih CC and Aboody KS: Identification of uPAR-positive chemoresistant cells in small cell lung cancer. PLoS One 2: e243, 2007.

27. LeBeau AM, Duriseti S, Murphy ST, Pepin F, Hann B, Gray JW, VanBrocklin HF and Craik CS: Targeting uPAR with antagonistic recombinant human antibodies in aggressive breast cancer. Cancer Res 73: 2070-2081, 2013.

28. Ma YY and Tao HQ: Role of urokinase plasminogen activator receptor in gastric cancer: A potential therapeutic target. Cancer Biother Radiopharm 27: 285-290, 2012.

29. Codony-Servat J, Verlicchi A and Rosell R: Cancer stem cells in small cell lung cancer. Transl Lung Cancer Res 5: 16-25, 2016.

30. Chiacchiera F, Matrone A, Ferrari E, Ingravallo G, Lo Sasso G, Murzilli S, Petruzzelli M, Salvatore L, Moschetta A and Simone C: p38alpha blockade inhibits colorectal cancer growth in vivo by inducing a switch from HIFlalpha- to FoxO-dependent transcription. Cell Death Differ 16: 1203-1214, 2009.

31. Gopinath S, Malla RR, Gondi CS, Alapati K, Fassett D, Klopfenstein JD, Dinh DH, Gujrati M and Rao JS: Co-depletion of cathepsin B and uPAR induces G0/G1 arrest in glioma via FOXO3a mediated p27 upregulation. PLoS One 5: e11668, 2010 . 